\title{
Renal Tuberculosis Mimicking Hydronephrosis
}

Key words: renal tuberculosis, MR urography, CT scan

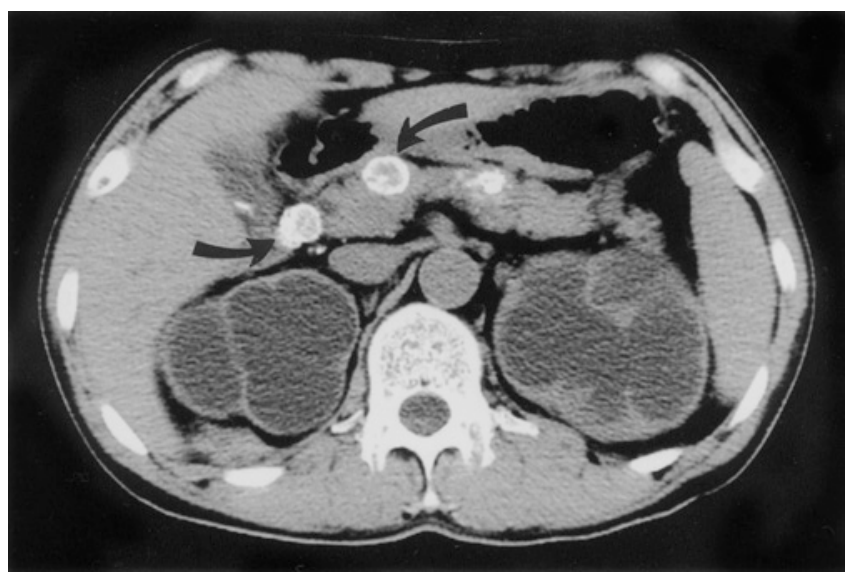

Figure 1. Axial non-contrast image of CT scan showing enlargement of both kidneys, cortical thinning with low-density, markedly dilated calices. Note calcification of lymph nodes surrounding the pancreas (arrows).

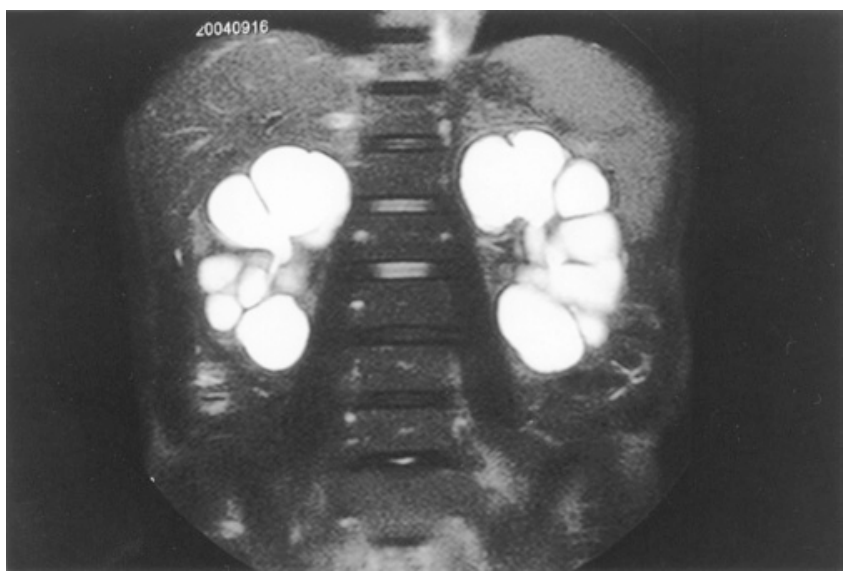

Figure 2. Coronal image of MR urography revealing multiple cyst-like calices that clearly showed stenosis and stricture of the caliceal infundibula.

A 43-year-old man has been on chronic hemodialysis for two years. Three years previously, he had recurrent episodes of dysuria and gross hematuria. At that time, Mycobacterium tuberculosis was isolated in a urine culture and he had received a therapy of antituberculous multiple drugs. During an annual check-up, screening of abdominal computer tomography (CT) was performed in June 2004. Axial non-contrast image of CT scan showed enlargement of both kidneys, cortical thinning with markedly dilated calices, which appeared to communicate with the renal pelvis (Fig. 1). CT findings seemed to be compatible with bilateral hydronephrosis. Furthermore, CT film showed calcification of lymph nodes surrounding the pancreas. But coronal image of magnetic resonance (MR) urography demonstrated multiple cyst-like calices with well-defined margins, representing hydronephrotic calices secondary to infundibular stricture and/or renal papillary necrosis (Fig. 2). The coronal image of MR urography is the most sensitive in showing caliectasis without renal pelvic dilatation.

Norihiko Matsumura, Kazuo Yamamoto*, Rina Hirohashi** and Satoru Kitano**

From the Department of Nephrology, *Cardiology and **Radiology, Saiseikai Suita Hospital, Suita

Received for publication February 2, 2005; Accepted for publication March 6, 2005

Reprint requests should be addressed to Dr. Norihiko Matsumura, the Department of Nephrology,

Saiseikai Suita Hospital, 1-2 Kawazono-cho, Suita, Osaka, 564-0013 